\title{
Diseño de un tanque de almacenamiento de agua caliente sanitaria, utilizando materiales de bajo costo
}

\author{
Design of a storage tank hot water, using inexpensive materials
}

\author{
Juan Alberto Mantilla, Mario Alexander Casallas \\ Facultad Tecnológica, Universidad Distrital Francisco José de Caldas, Bogotá, Colombia \\ jamantillalecorreo.udistrital.edu.co \\ maacasallasrecorreo.udistrital.edu.co
}

\begin{abstract}
Resumen- En este artículo se presenta el proceso de diseño y construcción de un tanque de almacenamiento de agua caliente sanitaria (ACS) para consumo doméstico. Además, se describe el análisis matemático, la selección de materiales tanto para el aislamiento como para el cuerpo del tanque, la simulación del comportamiento hidrostático, el proceso de construcción con base en la norma Asme sección VIII y las pruebas de funcionamiento del mismo.

Este trabajo hace parte de un proyecto de investigación orientado al diseño y construcción de calentadores solares a partir de materiales reutilizables, realizado por el semillero de investigación SEA, perteneciente al grupo de investigación en energías alternativas Gieaud, de la Universidad Distrital Francisco José de Caldas.

El calentador solar y el tanque se encuentran montados en la terraza del gimnasio ubicado en la Facultad Tecnológica y se le realizaron pruebas para evaluar la eficiencia en la conservación de la temperatura y actualmente se continúa con pruebas adicionales que permitan observar mejor el desempeño del tanque.
\end{abstract}

Palabras clave - Tanque de almacenamiento, Aislamiento térmico, Norma Asme, Transferencia de calor, Recipiente a presión.

Abstract-This article describes the process of design and construction of a storage tank hot water (DHW) for domestic consumption is presented. In addition, mathematical analysis is described, the selection of materials for both insulation and the body of the tank, hydrostatic simulation behavior, the construction process based on ASME Section VIII and testing operation.

This work is part of a research project aimed at designing and construction of solar heaters from reusable materials by the hotbed of research SEA belonging to the group of alternative energy research GIEAUD District University of Francisco José de Caldas.

The solar heater and the tank are mounted on the roof of the gym located in the Faculty of Technology. Tests were performed to evaluate the efficiency in maintenance of the temperature and currently continues with additional tests to better observe the performance of the tank.
Key Word - Thermal insulation, ASME, Heat transfer, Pressure vessel.

\section{INTRODUCCIÓN}

El calentamiento de agua para el consumo doméstico, o la producción de agua caliente sanitaria (ACS) por medio de la energía solar, es una alternativa muy importante para sustituir los calentadores tradicionales (a gas, eléctricos, etc.) debido al ahorro energético que éste representa, al permitir el reemplazo de los combustibles fósiles o la electricidad para lograr este propósito.

Un calentador solar de ACS, construido con materiales reutilizables, presenta ciertas ventajas comparadas con los calentadores solares de placa plana o de tubos al vacío, como su bajo costo, pero también implica inconvenientes, como su baja eficiencia por las pérdidas de calor que se presentan en el tanque de almacenamiento del ACS, principalmente en las horas nocturnas, entre otros aspectos. Por esta razón y por seguridad, se requiere de la construcción de un tanque que cumpla con normas internacionales, como se regula en Asme [1]

El presente trabajo hace parte de un proyecto de investigación general, que se ha venido desarrollando al interior del semillero de investigación en energías alternativas de la Universidad Distrital (SEA), el cual consiste en el diseño y construcción de un calentador solar usando material reciclable o de bajo costo. Para esto se ha trabajado en dos frentes: uno consistente en diseñar y construir el colector solar que produzca ACS a mínimo $40^{\circ} \mathrm{C}$ con la radiación solar promedio de la ciudad de Bogotá, y el otro dedicado al diseño y construcción del tanque de almacenamiento.

Este proyecto se ha desarrollado con el objetivo de

- Diseñar y construir un tanque de almacenamiento de ACS para el calentador solar con mejor rendimiento construido hasta el momento por los estudiantes del semillero SEA y el grupo de investigación Gieaud, que corresponde a un 
calentador solar construido con tubería galvanizada en paralelo y envases Pet translúcidos para 120 litros de agua, con lineamientos de la Norma Asme sección VIII división 1 Recipientes a presión.

\section{Diseño y construcción del tanque}

En esta sección se detalla el procedimiento llevado a cabo para el diseño y construcción del tanque, además del análisis y selección del aislante térmico y la evaluación del rendimiento del mismo.

A. Determinación de las dimensiones del tanque y simulación

Para el desarrollo de este ítem de diseño, se ha tomado como referencia las 'Reglas para la construcción de recipientes a presión' de la Norma Asme [2], de donde se brindan los parámetros necesarios para determinar las dimensiones del tanque, como la longitud, el diámetro, la presión de diseño, el espesor y tipo de material, entre otros.

Las especificaciones para este tanque son:

- Capacidad de almacenamiento: $120 \mathrm{~L}\left(4,2372 \mathrm{ft}^{3}\right)$, que va a ser utilizada para uso doméstico y cubrirá la necesidad de una familia promedio de 4 personas.

- Temperatura de trabajo: $60{ }^{\circ} \mathrm{C}\left(140{ }^{\circ} \mathrm{F}\right)$. Aunque la temperatura de trabajo mínima es de $40{ }^{\circ} \mathrm{C}$, se adopta un valor de temperatura mayor, contemplando un factor de seguridad, que en este caso es de 1,5.

- Presión de operación: 0,1 Mpa (14,5 psi).

- Presión de diseño: 0,2 Mpa (29 psi).

El material que se trabajará es Acero 1020, este es aceptado por la norma en mención, con algunas modificaciones sugeridas por la misma [2], cuyas propiedades mecánicas se citan a continuación:

- Resistencia a la fluencia $\mathrm{Sy}=200 \mathrm{Mpa}(29 \mathrm{Ksi})$.

- Esfuerzo último a la tensión Sut=414 Mpa (60 Ksi).

- Módulo de elasticidad E= $200 \mathrm{Gpa}$ $\left(29 \times 10^{6} \mathrm{psi}\right)$

- coeficiente de conductividad térmica $\mathrm{k}=41 \mathrm{~W} / \mathrm{m} * \mathrm{~K}$

Para el análisis de las soladuras, se siguen los lineamientos de la sección UW, en la que la eficiencia de la soldadura está en función de la inspección de la misma (tintas penetrantes o rayos $\mathrm{x}$ ) donde el valor máximo es 1 . Para este caso, el valor de la eficiencia es 0,7 porque no se cuenta con inspecciones certificadas tanto en las tapas como en el cuerpo [3].
B. Presión de diseño

El valor que se usa en las ecuaciones para el cálculo de las partes del tanque es el siguiente.

- $\quad$ Para Po $>300$ psi (2,07MPa), entonces $\mathrm{P}=1,1 \mathrm{Po}$

- $\quad$ Para Po < 300 psi (2,07MPa), entonces $\mathrm{P}=\mathrm{Po}+30$ psi; donde $\mathrm{P}$ es la presión de diseño y Po la presión de operación. Entonces, para este caso, la presión de diseño $\mathrm{Po}=30$ psi $(0,21 \mathrm{MPa})$ y la presión de operación $\mathrm{P}=60 \mathrm{psi}(0,41 \mathrm{MPa})$

\section{Diseño óptimo del tanque}

Para el diseño del tanque, se establece la relación del diámetro con la longitud del mismo. La ecuación que se emplea es la siguiente.

$$
F=\frac{P}{(C S E)}
$$

donde

- $\mathrm{P}=$ presión de diseño (psi)

- $\mathrm{C}=$ corrosión máxima permitida (in). La corrosión máxima permitida no puede exceder 1/6 del espesor del material. [4]

- $\quad \mathrm{S}=$ valor del esfuerzo del material (psi)

- $\mathrm{E}=$ eficiencia de la soldadura (adimensional).

- $\mathrm{F}=$ relación entre el diámetro y longitud del recipiente (adimensional).

$$
\begin{gathered}
F=\frac{60 \text { psi }}{\left.\left(\frac{1}{24}\right) * 50 * 10^{3} \text { psi } * 0,7\right)} \\
\mathrm{F}=0,4114
\end{gathered}
$$

Ahora, con el valor del factor $\mathrm{F}=0,4114$ y el volumen del recipiente $\mathrm{V}=4,23 \mathrm{ft}^{3}\left(0,12 \mathrm{~m}^{3}\right)$, se busca el diámetro del tanque en a la siguiente figura:

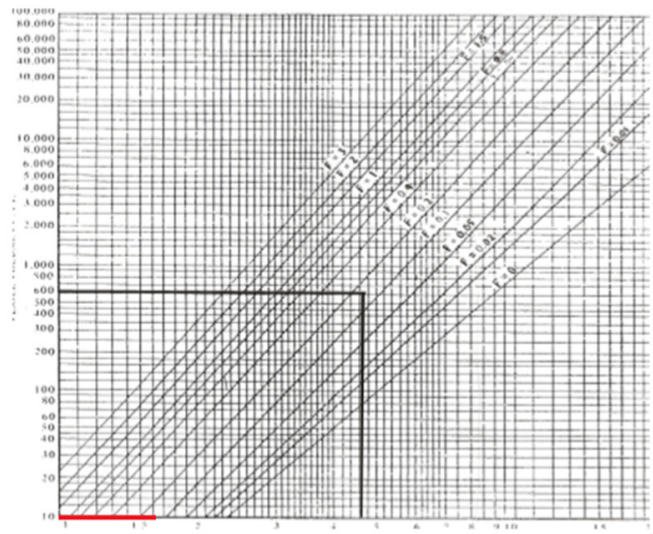


Figura 1. Determinación grafica diámetro exterior del tanque (ver anexo 2 tabla de obtención de diámetros tanque) [5]

Según la figura 1, el diámetro exterior optimo del tanque es de $1,3 \mathrm{ft}$ o $400 \mathrm{~mm}$ y, con este valor, se calcula la longitud óptima de acuerdo con la ecuación 2 .

$$
L=\frac{4 V}{\pi D^{2}} \quad(\mathbf{2})
$$

Donde:

- $\quad \mathrm{L}=$ longitud optima del recipiente (mm o inch)

- $\quad \mathrm{V}=$ volumen de trabajo $\left(\mathrm{ft}^{3} \mathrm{o} \mathrm{L}\right)$

- $\mathrm{D}=$ diámetro exterior optimo ( $\mathrm{mm}$ o inch).

Entonces:

$$
\begin{aligned}
& L=\frac{4\left(4,2372 f t^{3}\right)}{\pi(1,3)^{2}} \\
& \mathrm{~L}=3.1 \mathrm{ft}(944 \mathrm{~mm})
\end{aligned}
$$

Una vez obtenidos los resultados de diámetro y longitud, se determina, en el Anexo 2 (memorias de cálculo cuerpo del tanque) el espesor de la lámina del tanque de 1/8 in, y el tipo de tapas laterales a utilizar (tapas elípticas) tal como se muestra en el Anexo 3 (planos tanque) las dimensiones finales para la construcción del mismo, basados bajo [6].

\section{Análisis hidrostático}

El propósito de este análisis es saber si el tanque diseñado va a soportar el peso del agua, además de la presión a la que va a estar sometido. También se busca establecer si el material escogido es el apropiado para que éste no vaya a sufrir algún deterioro o no cumpla con los requerimientos de diseño. El análisis se realiza mediante uso del software Ansys en el que se pretende encontrar los rangos de esfuerzos y deformaciones que presenta el tanque, así como determinar si cumple o no con las especificaciones de diseño.

En la figuras 2 y 3 se aprecian los esfuerzos y deformaciones a los cuales está sometido el tanque bajo las condiciones de diseño.

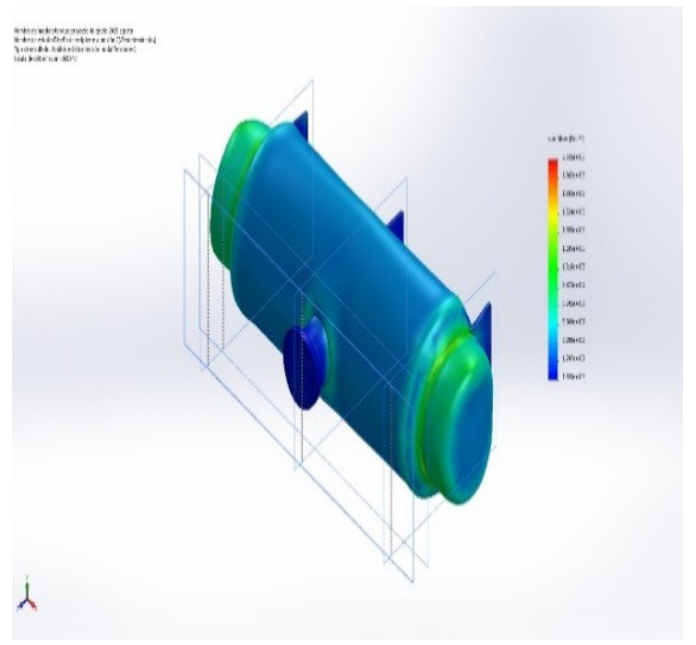

Figura 2. Comportamiento del material del tanque por esfuerzos

Con una presión de 30 psi (0,21 $\mathrm{MPa})$ y una temperatura de operación de $60{ }^{\circ} \mathrm{C}$, la simulación hecha por el software Ansys muestra que la deformación máxima es de 8,4 e-3 pulgadas $(0,2 \mathrm{~mm})$ y que el esfuerzo máximo está en el orden de 3,6 psi $(24,8 \mathrm{KPa})$, lo cual garantiza que el recipiente no va a fallar porque no excede la presión de diseño (30 psi). Por lo tanto, el espesor de la lámina del tanque es apropiado para realizar y llevar a cabo su función. En efecto, el espesor $3 \mathrm{~mm}$ es apropiado para su construcción.

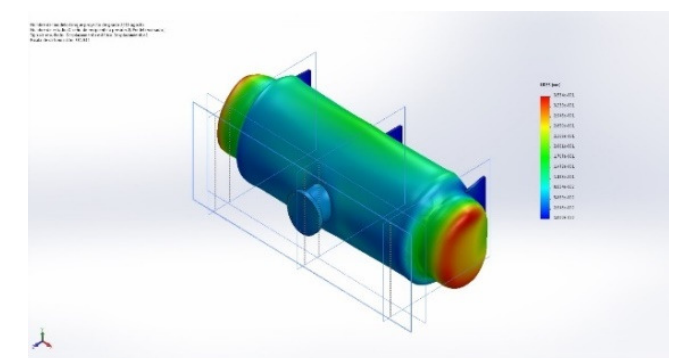

Figura 3. Comportamiento del material del tanque por deformación.

El software Ansys muestra, de manera exagerada, dónde es posible que falle el elemento. Una vez realizadas estas simulaciones, se procede a determinar cuál es el mejor tipo de aislante para el cuerpo del tanque, el cual conservará la temperatura mínima del ACS para su consumo.

\section{Determinación del tipo de aislante}

Los materiales preseleccionados para determinar el de mejor comportamiento son el papel corrugado, el cartón, el poliestireno expandido y la lana de vidrio. La selección se debe a que son de fácil obtención, es decir, se pueden encontrar en sitios habituales como viviendas, empresas, centro de reutilización de materiales o universidades, y su valor de adquisición es relativamente bajo en condiciones iniciales del diseño. A continuación se muestra proceso seguido para la selección del tipo de aislante. 


\section{Condiciones iniciales}

Al desarrollo del cálculo para determinar cuál de los materiales preseleccionados ofrece las mejores condiciones de aislamiento térmico, se siguen las recomendaciones del ingeniero Cengel, que recomienda evaluar el radio crítico de aislamiento, la razón de pérdida de calor, el espesor del aislante $\mathrm{y}$, finalmente, la transferencia de calor con el aislante [7].

En la figura 4 se muestran las dimensiones básicas con las cuales se realizan los diferentes cálculos.

\section{L: Longitud 0,944 m}

$\boldsymbol{r}_{\mathbf{1}}$ : Diámetro: $0.200 \mathrm{~m}$

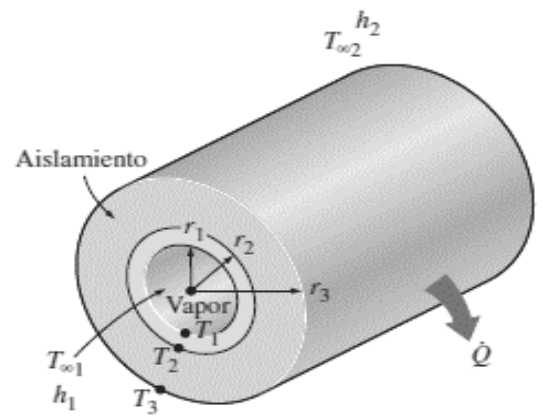

Figura 4. Modelo cálculo de aislante térmico.

$r_{1}$ : radio interior del tanque

$r_{2}$ : radio exterior del tanque

$r_{3}$ : radio exterior del aislamiento

En la Tabla 1 se muestran los valores de coeficiente de conductividad térmica y calor específico de los materiales aislantes preseleccionados.

\begin{tabular}{|c|c|c|}
\hline MATERIAL & $\mathbf{K}(\mathrm{W} /(\mathrm{m} \cdot \mathrm{K}))$ & $\mathbf{C p}(\mathbf{K J} /(\mathrm{kg} \cdot \mathrm{K}))$ \\
\hline Papel corrugado & 0,039 & 1,9 \\
\hline Cartón & 0,03 & 1,75 \\
\hline $\begin{array}{c}\text { Poliestireno } \\
\text { expandido }\end{array}$ & 0,040 & 1,2 \\
\hline Lana de vidrio & 0,044 & 0,96 \\
\hline
\end{tabular}

Tabla 1. Coeficientes de conductividad térmica y calor específico materiales. [7]
2. Procedimiento para calcular el radio crítico de aislamiento

El radio crítico de aislamiento para un cuerpo cilíndrico esta denotado por:

$$
r_{\text {C, cilindro }}=\frac{k}{\mathrm{~h}}
$$

Dónde:

$\mathbf{r}_{\mathbf{c}}=$ radio crítico $(\mathrm{m})$

$\mathbf{k}=$ coeficiente de conductividad térmica $\left(\mathrm{W} / \mathrm{m}{ }^{\circ} \mathrm{C}\right)$

$\mathbf{h}=$ coeficiente de convección térmica $\left(\mathrm{W} / \mathrm{m}^{2}{ }^{\circ} \mathrm{C}\right)$

3. Procedimiento para obtener la razón de pérdida de calor sin aislante

En la ecuación 4 se muestra la pérdida de calor:

$$
\begin{aligned}
& \dot{Q}=h_{0} A_{0}\left(T_{\infty 1}-T_{\infty 2}\right) \\
& A_{0}=\pi \mathrm{DL}
\end{aligned}
$$

Dónde:

$\mathbf{h}_{\mathbf{o}}$ : Coeficiente combinado de transferencia de calor en el tubo $\boldsymbol{A}_{\mathbf{0}}$ : área exterior cuerpo del tanque

$\mathbf{T}_{\mathbf{S}}$ : temperatura superficie Exterior del tanque sin aislante $\mathbf{T}_{\text {air }}$ : temperatura aire.

Por medio de este modelo se determina la cantidad de energía que se pierde en el tanque.

\section{Procedimiento para calcular el espesor del aislante}

Las ecuaciones 6,7 y 8 , respectivamente, muestran la pérdida de calor en términos generales, en el tanque sin aislamiento y el cálculo del espesor, teniendo una pérdida de 565,5 w sin aislante y fijando como parámetro una pérdida máxima de 200 w equivalente al $66 \%$.

$$
\begin{gathered}
\dot{Q}=\frac{T_{S}-T_{\text {air }}}{\text { Rs-Rais }} \\
\dot{Q}=\frac{T_{\infty 1}-T_{\infty 2}}{\frac{1}{h_{0} A_{0}}-\frac{\left(L n^{\left.r_{3} / r_{2}\right)}\right.}{2 \pi K L}} \\
r_{3}-r_{2}=\mathrm{X}
\end{gathered}
$$

5. Procedimiento para determinar la pérdida de calor con el aislante respectivo

A continuación se describen las ecuaciones para determinar cuál es la transferencia de calor correspondiente del tanque al medio, teniendo en cuenta el espesor de cada tipo de material aislante. 
Los valores de $\mathrm{R}$, en la ecuación 6, corresponden a las resistencias térmicas que tiene cada material para transferir calor.

Los valores de las resistencias térmicas se calculan de la siguiente manera:

$$
\begin{aligned}
& R \text { conv }, 1=\frac{1}{h_{1} \mathrm{~A}} \quad\left({ }^{\circ} \mathrm{C} / \mathrm{w}\right) \\
& R \text { tubo }=\frac{\ln \left(\frac{r_{2}}{r_{1}}\right)}{2 \pi k_{1} \mathrm{~L}} \quad\left({ }^{\circ} \mathrm{C} / \mathrm{w}\right) \\
& R \text { aislamiento }=\frac{\ln \left(\frac{r_{3}}{r_{2}}\right)}{2 \pi k_{2} \mathrm{~L}} \quad\left({ }^{\circ} \mathrm{C} / \mathrm{w}\right) \\
& \text { Rconv } 2=\frac{1}{h_{2} A_{3}} \quad\left({ }^{\circ} \mathrm{C} / \mathrm{w}\right) \\
& R \text { total }=R_{\text {conv }, 1}+R_{\text {tubo }}+R_{\text {ais }+} R_{\text {conv }, 2}
\end{aligned}
$$

Donde:

$\mathbf{T}_{\infty 1}$ : Temperatura del ACS $\left(40^{\circ} \mathrm{C}\right)$

$\mathbf{T}_{\infty \mathbf{2}}$ Temperatura ambiente $\left(10^{\circ} \mathrm{C}\right)$

$\mathbf{h}_{1}, \mathbf{h}_{2}$ : Coeficiente de conveccion del tanque y amb.

$\boldsymbol{A}_{\mathbf{1}}, \mathbf{A}_{\mathbf{3}}$ : Area superficie del tanque y aislante.

$\boldsymbol{k}_{\mathbf{1}}, \mathbf{k}_{\mathbf{2}}$ : Coef. Transferencia de calor tanque y aislante.

La Tabla 2 muestra los resultados obtenidos a partir de la ecuación 3 hasta la ecuación $13 \mathrm{y}$, con estos valores, se determina el aislante térmico con el cual se va a recubrir el tanque.

\begin{tabular}{|c|c|c|c|c|}
\hline MATERIAL & $\mathbf{r c}(\mathbf{c m})$ & $\begin{array}{c}Q(\mathrm{w}) \\
\text { Sin } \\
\text { Ais }\end{array}$ & $r_{3}(\mathrm{~cm})$ & $\begin{array}{c}\mathrm{Q}(\mathrm{w}) \\
\text { aislante }\end{array}$ \\
\hline $\begin{array}{c}\text { Papel } \\
\text { corrugado }\end{array}$ & 0,195 & 565,5 & 11,21 & 200 \\
\hline Cartón & 0,15 & 565,5 & 9,75 & 200 \\
\hline $\begin{array}{c}\text { Poliestireno } \\
\text { expandido }\end{array}$ & 0,2 & 565,5 & 7,13 & 200 \\
\hline $\begin{array}{c}\text { Lana de } \\
\text { vidrio }\end{array}$ & $\mathbf{0 , 2 2}$ & $\mathbf{5 6 5 , 5}$ & $\mathbf{5 , 5}$ & $\mathbf{2 0 0}$ \\
\hline \multicolumn{5}{|c}{ Tabla 2 Resultados mejor aislante. }
\end{tabular}

El radio crítico arroja valores muchos menores comparados con el radio 1 del tanque. Por eso, este valor no tiene relevancia en el proceso de escoger el espesor de los aislantes ya que el radio 3 siempre será mayor al radio crítico de diseño.

Los datos obtenidos arrojan que el mejor material aislante para recubrir el tanque es la lana de vidrio, con un espesor de pared mínimo de $5,5 \mathrm{~cm}$ de acuerdo con el parámetro de pérdida de calor fijado $(200 \mathrm{w})$.
En la figura 5 se aprecia el aspecto físico de cómo queda finalmente el tanque después de seleccionar el aislante apropiado y su recubrimiento metálico. Las dimensiones finales son:

- Diámetro con aislante $=510 \mathrm{~mm}$

- $\quad$ Longitud con aislante $=951 \mathrm{~mm}$

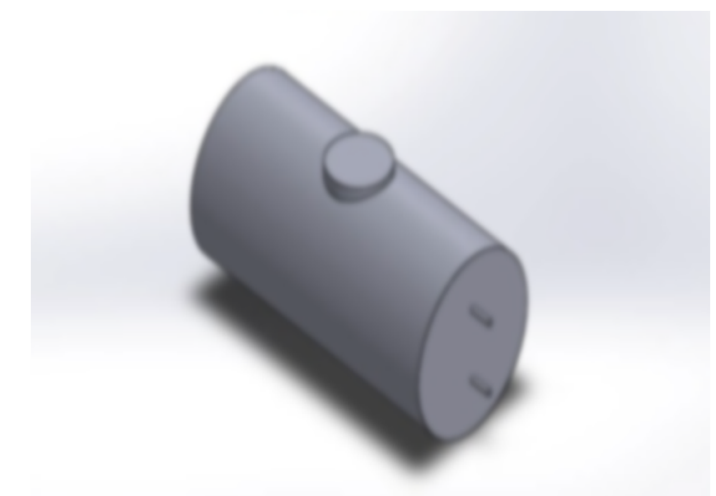

Figura 5. Tanque con coraza y aislante

E. Construcción y montaje del tanque al colector solar

Una vez se tienen todos los criterios de fabricación (tipo de aislante, dimensiones del cuerpo y posición), se ha procedido a construir y posteriormente ensamblar el tanque al captador solar escogido, (calentador en envases PET traslucido, con tubería galvanizada en paralelo) en la terraza del gimnasio de la Facultad Tecnológica de la Universidad Distrital Francisco José de Caldas.

Desde la figura 7 hasta la 10, se muestra un registro fotográfico del proceso de fabricación, pasando por la construcción del tanque, la unión del recubrimiento térmico, el sellamiento del tanque con su aislamiento y el montaje para sus respectivas pruebas.

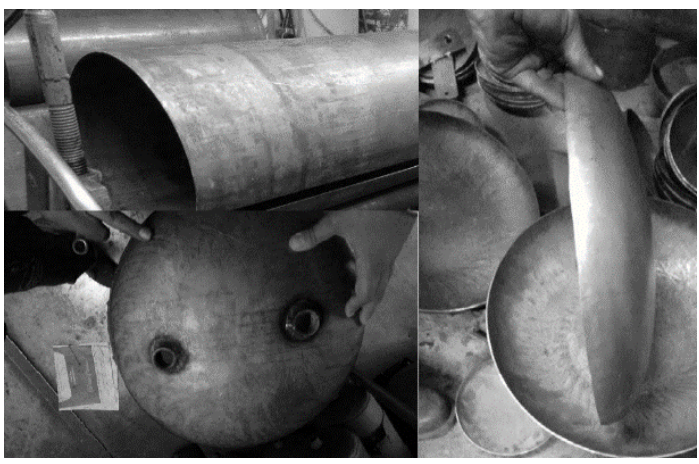

Figura 6. Fabricación tanque.

En la figura 6 se muestra el proceso de rolado y abombado de tapas. Se debe tener en cuenta las uniones soldadas para que así 
mismo no haya fugas y el funcionamiento del tanque sea el adecuado.

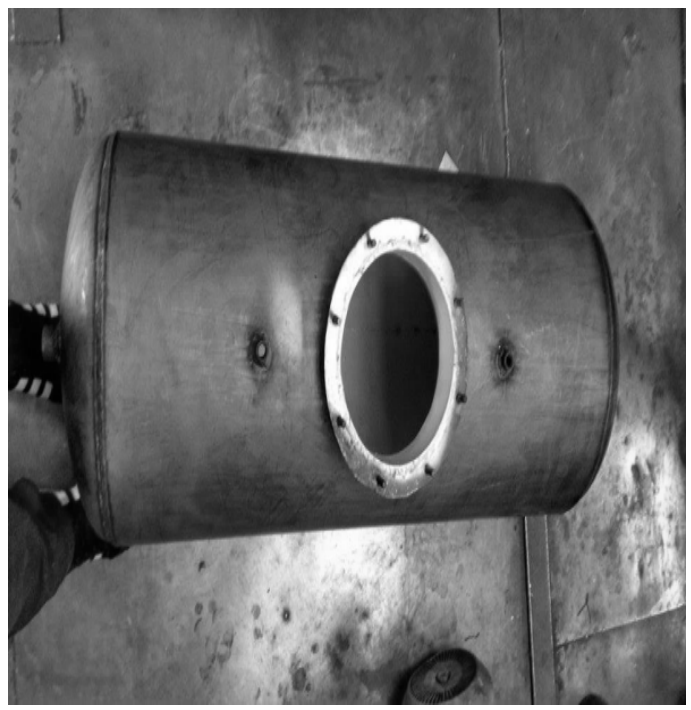

Figura 7. Cuerpo tanque.

En la figura 7, el cuerpo del tanque presenta un acceso de limpieza o handhole para realizar mantenimiento general y realizar futuras inspecciones de desgaste del material por corrosión. Según norma que se sigue [6].

Las figura 8 y la figura 9 muestra el recubrimiento de la lana de vidrio y su coraza protectora en lámina de acero calibre 14. Con esto, se garantiza que no queden espacios sin cubrir y queden en contacto directo con la intemperie.

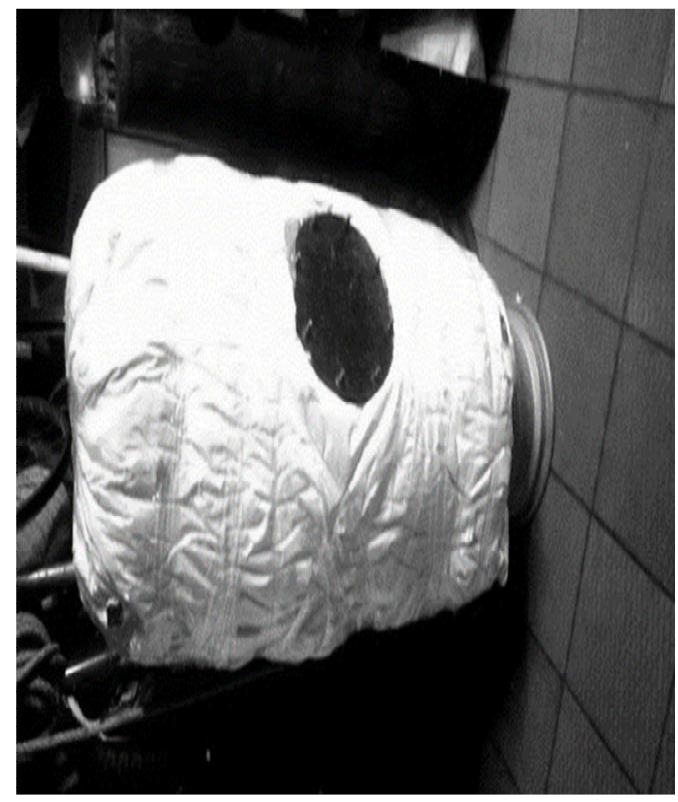

Figura 8. Montaje aislante (lana de vidrio)

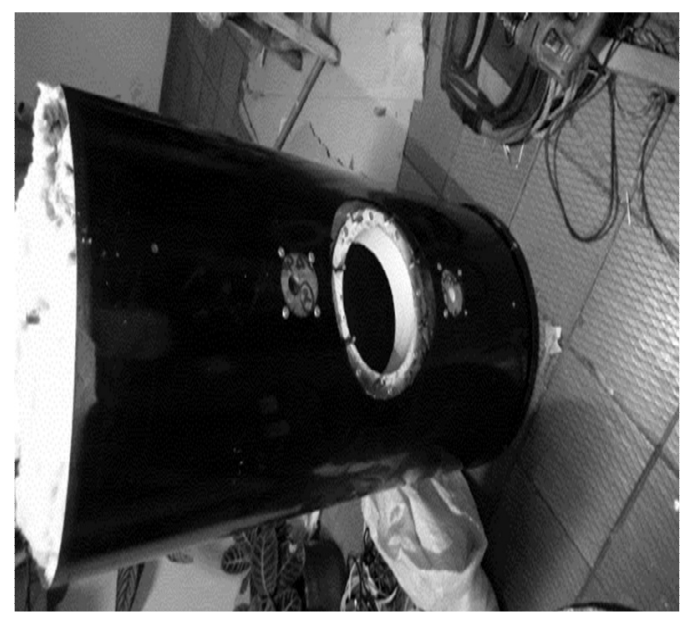

Figura 9. Ubicación carcasa y tapas laterales.

En la figura 10 se muestran tanto el tanque como el captador fijos en la estructura que previamente se ha dispuesto para este fin. Esta estructura está construida a partir de un ángulo estructural en L ASTM A36 de 2" x 3/8". El calentador se instala con la instrumentación necesaria para realizar las pruebas de funcionamiento: 3 termómetros de escala (-10 a 110 $\left.{ }^{\circ} \mathrm{C}\right)$ o $\left(20\right.$ a $\left.220{ }^{\circ} \mathrm{F}\right)$, que cumplen con la norma de EN-13190 [8], ubicados a la entrada del tanque, salida del colector y salida del tanque agua caliente; un manómetro y válvula de alivio para garantizar la seguridad del dispositivo al ser operado.

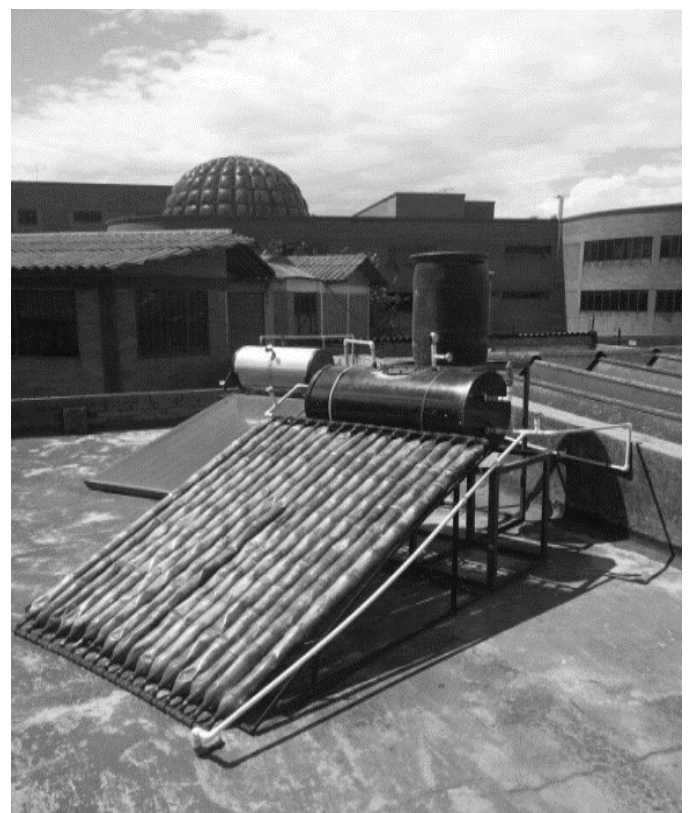

Figura 10.Ensamble, tanque, captador solar, instrumentos y accesorios para su correcto funcionamiento.

F. Prueba de fugas tanque captador solar

Una vez realizado el respectivo ensamble, se deja el tanque con agua un tiempo de 3 días esto con el fin de verificar que no 
posea algún tipo de fugas que podría repercutir en el buen funcionamiento del calentador solar, además de verificar que el captador solar este en buen estado y este no presente ninguna daño o deterioro entre sus componentes.

III. Ensayo del rendimiento del tanque en la conservación de la temperatura

A continuación, se menciona el procedimiento de cómo se realizaron las pruebas para verificar qué tan eficiente es el tanque, tomando el procedimiento, la obtención de datos y posteriormente su interpretación.

\section{A. Descripción del ensayo}

Una vez realizado el ensamblaje del tanque con el captador solar y elaborado la verificación de cero fugas, se procedió a tomar datos de temperatura y presión durante una semana (contemplada desde el 25 de marzo del 2015 al 1 de abril del 2015), teniendo tres horas establecidas: en la mañana a las 7:30 a. m., mediodía $12 \mathrm{~m}$., y tarde 5:30 p. m. con el fin de corroborar y realizar un consolidado de los datos para verificar si la construcción del tanque y el procedimiento que se llevó a cabo corresponden con el fin último, esto es, mantener la temperatura del agua más elevada para consumo doméstico.

\section{B. Datos obtenidos}

Los datos observados son los días en los que se realizó el ensayo que comprende el periodo desde 25 de marzo al $1^{\circ}$ de abril del 2015, donde se establece la temperatura ambiente del día y la presión manométrica registrada por el instrumento instalado.

\begin{tabular}{|c|c|c|c|c|c|c|c|c|}
\hline \multirow{3}{*}{ DIA } & \multicolumn{7}{|c|}{ TEMPERATURA } & \multirow{3}{*}{ Pm } \\
\hline & \multicolumn{2}{|c|}{$\begin{array}{l}7: 30 \\
\text { a.m. }\end{array}$} & \multicolumn{2}{|c|}{ 12:00 m. } & \multicolumn{2}{|c|}{ 5:30 p.m. } & \multirow{2}{*}{ Ta } & \\
\hline & in & out & In & out & In & out & & \\
\hline día 1 & $\begin{array}{l}3 \\
3\end{array}$ & 30 & 44 & 41 & 36 & 33 & 16 & 3,8 \\
\hline día 2 & $\begin{array}{l}2 \\
8\end{array}$ & 25 & 43 & 40 & 34 & 31 & 14 & 3,8 \\
\hline día 3 & $\begin{array}{l}3 \\
0\end{array}$ & 27 & 44 & 41 & 33 & 30 & 13,6 & 3,8 \\
\hline día 4 & $\begin{array}{l}2 \\
9\end{array}$ & 26 & 43 & 40 & 36 & 33 & 17 & 3,8 \\
\hline día 5 & $\begin{array}{l}3 \\
2\end{array}$ & 29 & 41 & 38 & 38 & 35 & 15 & 3,8 \\
\hline día 6 & \begin{tabular}{|l|}
2 \\
8
\end{tabular} & 25 & 42 & 39 & 35 & 32 & 13,5 & 3,8 \\
\hline día 7 & \begin{tabular}{|l|}
3 \\
1 \\
\end{tabular} & 29 & 45 & 42 & 33 & 30 & 14 & 3,8 \\
\hline día 8 & \begin{tabular}{|l|}
3 \\
0
\end{tabular} & 31 & 43 & 40 & 37 & 34 & 16 & 3,8 \\
\hline
\end{tabular}

Tabla 2. Datos obtenidos experimentalmente.

\section{Análisis de resultados}

En las gráficas 1 y 2, se evidencia la variación de temperatura durante los días evaluados, teniendo en cuenta la franja horaria en la que fueron tomados los datos (7:30 a. m., 12 m., 5:30 p. m.). Se observó que la temperatura más alta obtenida es a las $12 \mathrm{~m}$., y que este efecto se produce a la entrada y salida del tanque. Por el contrario, las temperaturas más bajas se registran a las 7:30 a. m. debido a las condiciones ambientales que a esa hora de la mañana se tienen.

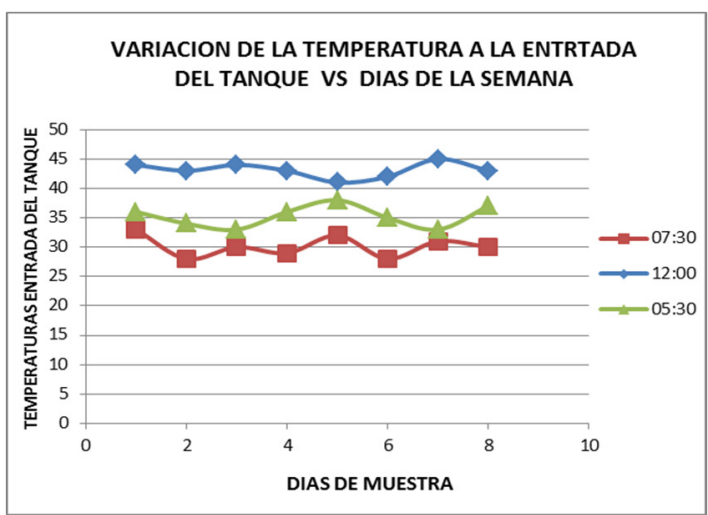

Gráfica 1. Comparación de temperatura a la entrada tanque de las tres horas establecidas.

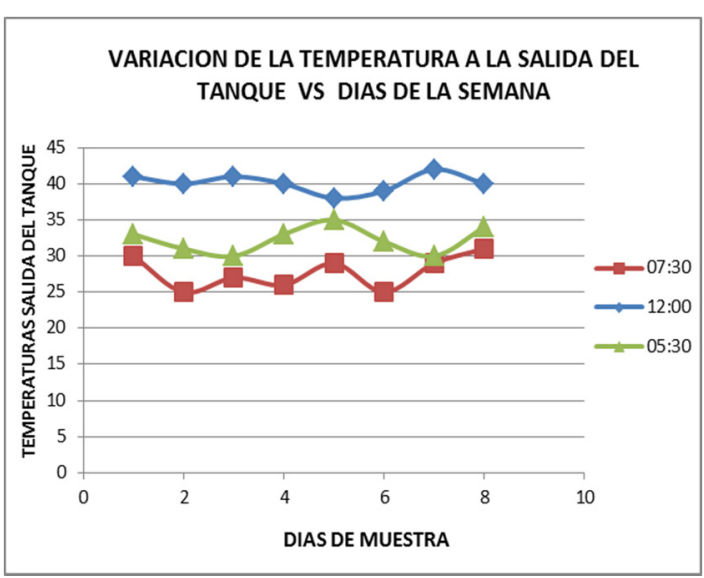

Grafica 2. Comparación de temperatura a la salida del tanque de las tres horas establecidas.

La franja horaria de 5:30 p. m. muestra un comportamiento promedio respecto a las otras temperaturas y franjas horarias evaluadas, y es la más estable en el proceso de recolección de datos.

Por lo anterior, el tanque presenta variaciones de temperatura menores a $5{ }^{\circ} \mathrm{C}$ a la entrada y salida del dispositivo, y las temperaturas del dispositivo entre las horas de la noche y la mañana están por encima de $\operatorname{los} 28{ }^{\circ} \mathrm{C}$, por lo que el tanque presenta un rendimiento del $72 \%$. 


\section{Costos}

En la Tabla 3, se mencionan los costos de ejecución del proyecto en términos generales.

\begin{tabular}{|c|c|c|c|c|c|}
\hline \multicolumn{6}{|c|}{ COSTOS TANQUE } \\
\hline Elemento & und & & costo unidad & & sto total \\
\hline $\begin{array}{l}\text { fabricación (comprende rolado } \\
\text { de lámina ,abombado de } \\
\text { tapas, soldadura, entradas de } \\
\text { tubería, handhole y pintura } \\
\text { epóxica) }\end{array}$ & 1 & $\$$ & 150.000 & $\$$ & 150.000 \\
\hline $\begin{array}{l}\text { Rolado tapa exterior, } \\
\text { aislamiento térmico, tapas } \\
\text { laterales con agujeros salida } \\
\text { tubería }\end{array}$ & 1 & $\$$ & 100.000 & $\$$ & 100.000 \\
\hline $\begin{array}{l}\text { accesorios(comprende lo que } \\
\text { es acoples tubería, tubería, } \\
\text { platinas de sujeción tanque, } \\
\text { tornillos, soldadura ,limpiador, } \\
\text { teflón ,silicona, brocas, } \\
\text { válvulas de salida, todo } \\
\text { manejado en tubería de } 1 / 2 " \text { ) }\end{array}$ & 1 & $\$$ & 120.000 & $\$$ & 120.000 \\
\hline $\begin{array}{l}\text { Instrumentación(comprende lo } \\
\text { que son termómetro, válvula } \\
\text { alivio de presión, manómetro) }\end{array}$ & 1 & $\$$ & 160.000 & $\$$ & 160.000 \\
\hline $\begin{array}{l}\text { pintura (acabado final)latas de } \\
850 \mathrm{ml}\end{array}$ & 2 & $\$$ & 8.000 & $\$$ & 16.000 \\
\hline transporte y otros & 1 & $\$$ & 25.000 & $\$$ & 25.000 \\
\hline & & & total & & 571.000 \\
\hline
\end{tabular}

Tabla 3. Costos ejecución proyecto.

De lo anterior, el costo neto de la construcción del tanque es de \$ 370.000 por lo que económicamente es factible para su posible comercialización. Los otros costos se deben a que el tanque debe tener pruebas de funcionamiento, pero dicha instrumentación no es vital para que el tanque pueda estar operando. El costo total es de $\$ 571.000$ y resulta más económico al compararlo con uno comercial está en promedio de $\$ 900.000$.

\section{Conclusiones}

- El captador seleccionado funcionó de manera adecuada debido a que la temperatura del agua entregada a la entrada del tanque tuvo un promedio de $41^{\circ} \mathrm{C}$; sin embargo, este valor sigue siendo bajo para las expectativas de operación.

- El tanque presentó un comportamiento óptimo debido a que no hubo disminución en la temperatura por encima de los $5^{\circ} \mathrm{C}$, importante sobre todo en la franja de la tarde a la mañana del siguiente día. El rendimiento del tanque es de $72 \%$, por lo cual se recomienda tomar más datos para poder obtener un mejor rendimiento del mismo.

- El tanque, a pesar de no tener inspecciones que afectan el factor de seguridad, cumplió con las pruebas hidrostáticas, porque las presiones al interior del recipiente estuvieron siempre dentro de los valores de diseño; adicionalmente el tanque no presento ningún tipo de fugas.

- Se evidencia que la lana de vidrio presenta una pérdida de calor mucho menor con respecto a los otros materiales aislantes escogidos; papel corrugado, cartón y poliestireno expandido, teniendo como espesor de material $5,5 \mathrm{~cm}$ con una pérdida máxima de calor de $200 \mathrm{w}$, que $66 \%$ de la pérdida de calor total sin aislante.

- Se debe tener en cuenta que este tanque funciona con un reservorio que en este caso es el sol y no cumple con las condiciones de fidelidad, es decir, cuando hay días opacos, lluviosos u otros el sistema no va a recibir las mismas cantidades de energía que cuando hay un día soleado.

- El valor del tanque construido es relativamente bajo con respecto a los que se encuentran en el mercado, obteniendo una disminución en costo del $37,5 \%$, además ningún tanque comercial cuenta con acceso o handhole para mantenimiento o limpieza del mismo; con lo cual da un valor agregado en su fabricación.

- Con la experiencia ganada en la construcción y elaboración del tanque y ensamble de captadores solares se concluye que es una buena opción seguir en la investigación de métodos más elaborados que permitan mejorar el funcionamiento de estos y se dé utilización a nuevos materiales para la fabricación de los colectores seguir con el buen aprovechamiento de la energía solar.

\section{Agradecimientos}

- A la universidad distrital francisco José de caldas por su valiosa colaboración en la adecuación de espacios y ejecución de los procesos educativos.

- Al semillero de energías alternativas (SEA) por su gran esfuerzo en el trabajo investigativo realizado hasta el momento sobre calentadores solares y aprovechamiento de energía solar.

- Al tutor, y director German López Martínez quien fue persona indispensable para llevar a cabo y culminar la etapa de investigación y desarrollo de dicho proyecto.

\section{Referencias}

[1] Asme, Sección VIII Div. 1, 2011.

[2] Asme, Sección II Div. 1, 2011.

[3] Asme, Sección VIII Div 1 UW, 2011.

[4] Asme, Sección VIII Div. 1 UG, 2011. 
[5] A. G. Mejía y C. A. Reyes, Memoria, cálculo, selección y analisis numérico de un repiente a presión de $600 \mathrm{ft} 3$ de capacidad, Mexico D.F., 2008.

[6] Asme, Seccion VIII Div. 2, 2011.

[7] Y. A. Celgel, Transferencia de Calor y Masa, Mexico : Mc graw Hill, 2007.

[8] EN-13190, (Mechanical temperature measuring instruments). 